\title{
THE SHOCK STRUCTURE IN CURRENT-CARRYING JETS
}

\author{
H. Lesch, S. Appl, M. Camenzind \\ Landessternwarte Königstuhl \\ 69 Heidelberg, FRG
}

We exploite the detailed calculations and observations of the earth bow-shock and suggest that similar collective plasma processes can account for the dissipation in hot spots of extragalactic radiosources. On the base of magnetohydrodynamic models for the origin and propagation of current carrying jets we show that in the terminal shock in hot spots lower hybrid-turbulence is excited. This turbulence driven by drifting electrons in the shock provides the anomalous conductivity required for current closure. Lower-hybrid turbulence is also generated by ion reflection in the upstream plasma. The kinetic energy of these ion beams is partially transformed into lower hybrid wave energy. These waves also determine the ratio of energies taken for plasma heating and particle acceleration. This is due to the fact that lower hybrid waves propagate almost perpendicular to the magnetic fields (large (small) wavenumbers perpendicular (parallel) to the magnetic field), their phase velocity parallel to the B-field are much larger than perpendicular to the field. Therefore electrons and ions are differently coupled to these waves. The resonant interactions of the upstream plasma with these waves leads to strong ion heating and electron acceleration. The ions reach a temperature $\left(T_{i} \simeq 510^{11} \mathrm{~K}\right)$. The electrons are accelerated up to relativistic energies $(\gamma \simeq 1300)$.

The model explains the ion post-shock temperature required by the jump conditions and the electron preacceleration necessary for efficient diffusive shock acceleration (Lesch, Appl, Camenzind, 1989).

\section{References}

Lesch, H., Appl, S., Camenzind, M. (1989) 'Collective plasma processes in extragalactic radio sources', Astron. Astrophys., (in press) 